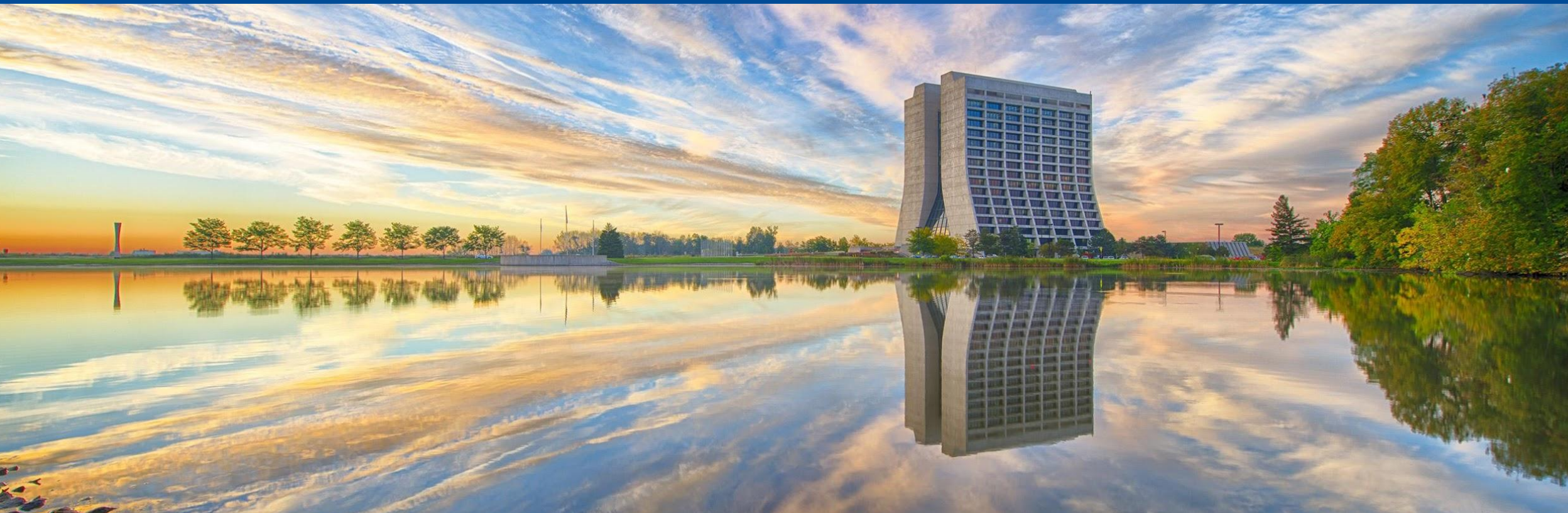

\title{
Performance Characterization of LCLS-II Superconducting Radiofrequency Cryomodules
}

Ruth Gregory

Lee Teng Internship Final Presentation 9 August, 2017

This manuscript has been authored by Fermi Research Alliance, LLC under Contract No. DEAC02-07CH11359 with the U.S. Department of Energy, Office of Science, Office of High Energy Physics
In partnership with:

MICHIGAN STATE

U N I V E R S I T Y
Illinois Accelerator Institute $W$

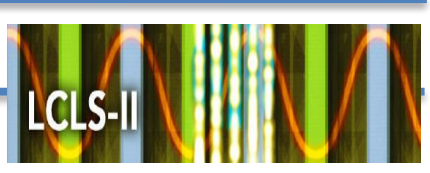




\section{Outline}

- Introduction

- LCLS-II and Fermilab's Contributions

- Motivation for Cryomodule Testing

- Analysis Methods

- Results

- Conclusion/Outlook 


\section{Linear Coherent Light Source (LCLS-II) and Fermilab's Contributions}

- LCLS II is a $2^{\text {nd }}$ generation $x$-ray free electron laser being constructed at SLAC National Accelerator Laboratory.

- Fermilab's contributions to LCLS-II include designing, assembling, and testing seventeen $1.3 \mathrm{GHz}$ and two 3.9 $\mathrm{GHz}$ cryomodules

- These cryomodules consist of eight nine-cell superconducting cavities.

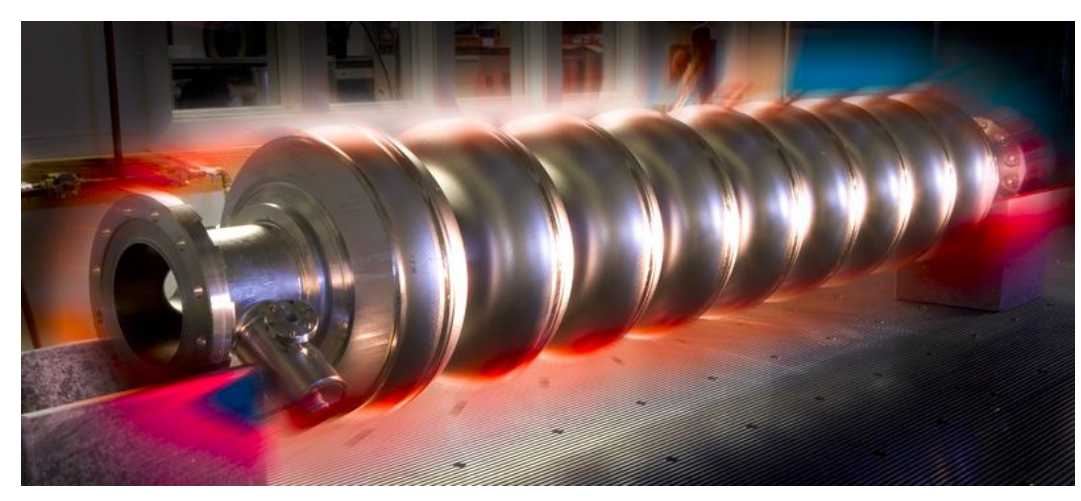

1.3 GHz Superconducting Radio Frequency (SRF) cavity

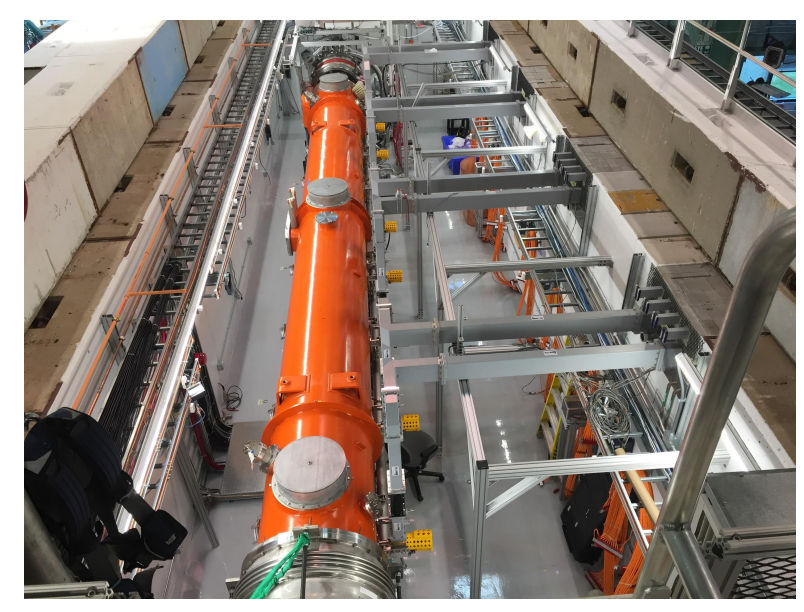

SRF cavity at Fermilab's cryomodule test stand 


\section{Motivation for Cryomodule Testing}

- Performance criteria are set for the level of field emission (radiation) and dark current produced by these cryomodules

- Assess which of the eight cavities produce the most (or any) radiation or dark current.

- Map out the radiation and dark current as a function of accelerating voltage.

- For each cavity determine:

- Peak radiation

- Cavity gradient $(\mathrm{MV} / \mathrm{m})$ where detectable radiation starts to appear

- This information will be included in the data travelers that will be sent to SLAC along with the cryomodules. 


\section{Analysis Methods}

- Cryomodule data including gradient and radiation levels is collected through Fermilab's Accelerator Control System (ACNET) archiving routines.

- A C++/ROOT program was developed to view and analyze the cryomodule data.

- This program generates:

- Plots of the data from each cryomodule device over time

- Plots of cavity voltage vs radiation

- Plots of dark current vs cavity voltage

- Data tables containing:

- Maximum values for each cryomodule device

- Peak radiation within any gradient range 


\section{Cryo Module Test Cave Radiation Detector Placement}

As of September 21, 2016

Chipmunks CMTS West Wall nos. $1-8$ correspond to locations $1,2,3,4,5,6,7,8$ :

G:RD3096,G:RD3097,G:RD3098, G:RD3099, G:RD3100, G:RD3101, G:RD3102, G:RD3103

CMTS South Gate

G:RD3107

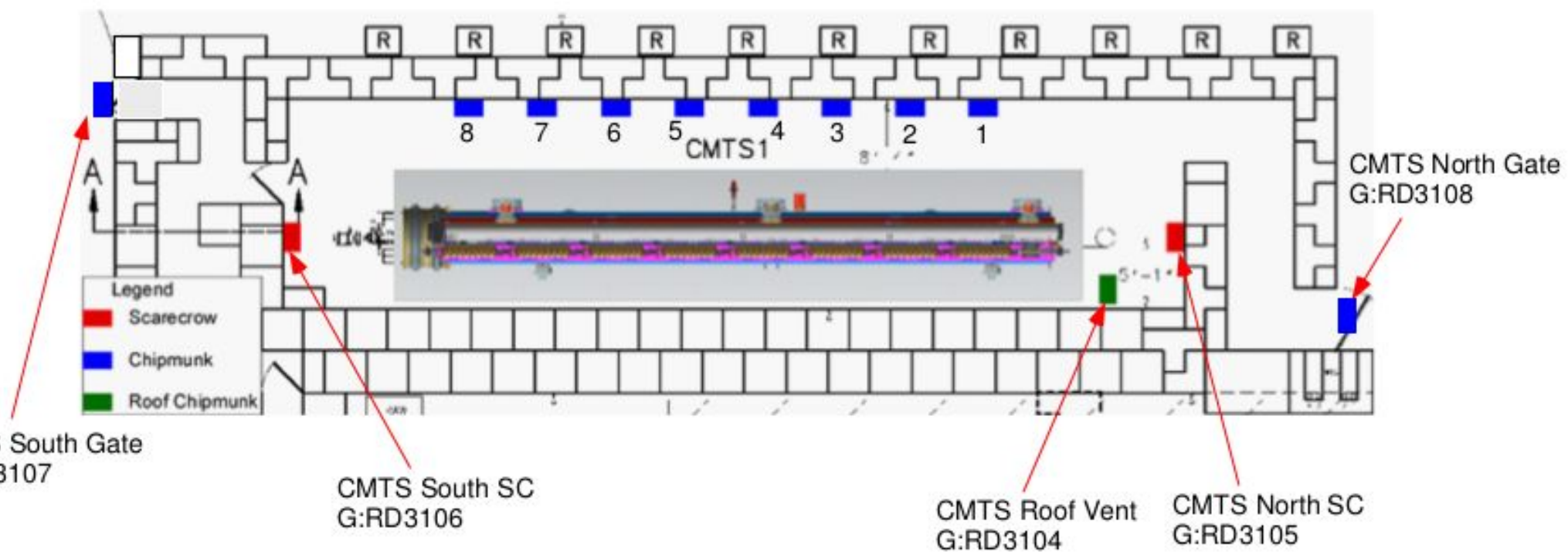

Drawing adapted from CMTS Preliminary Shielding Assessment v.4 Anthony F Leveling 12/4/2014 


\section{Results}

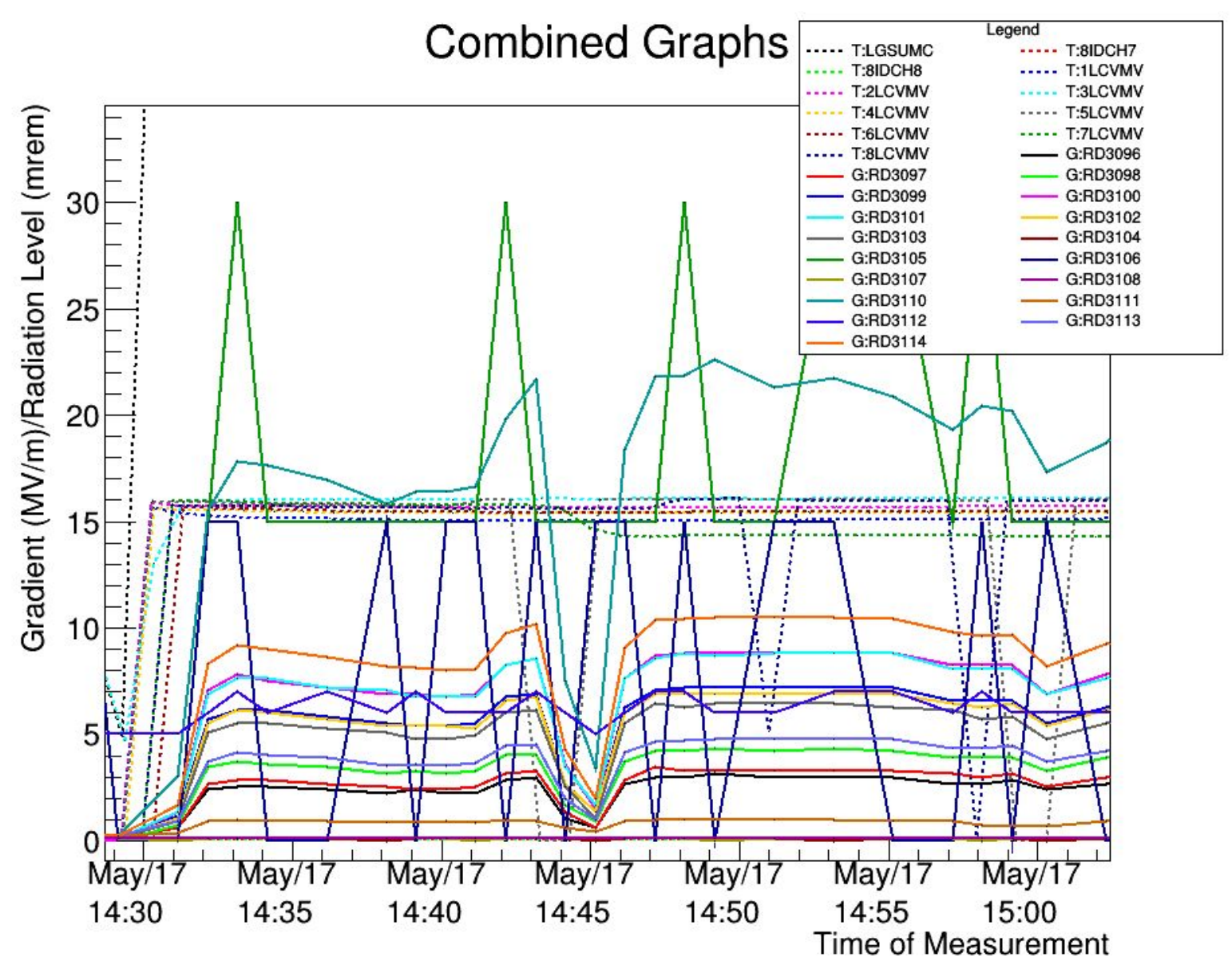

This plot shows the data from each cryomodule device plotted over time. The voltage cavity data has $\mathrm{y}$-axis units of $\mathrm{MV} / \mathrm{m}$, and is plotted with dotted lines. The radiation detector data has y-axis units of mrem, and is plotted with solid lines. 


\section{Results (cont.)}
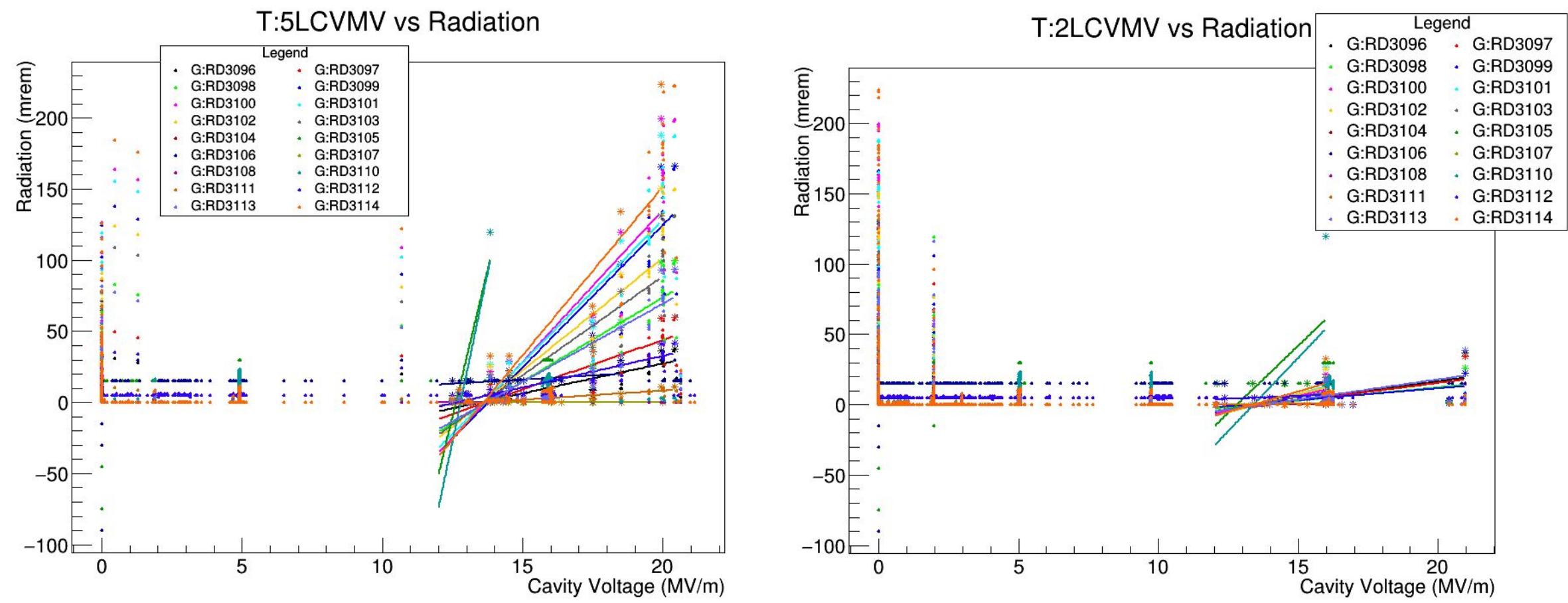

- These plots show the radiation levels for various radiation detectors plotted over the gradient for voltage cavities 5 (left) and 2 (right).

- The lines are linear fits of the data points in the range of $12 \mathrm{MV} / \mathrm{m}$ to the cavity voltage corresponding to the maximum radiation level for the given radiation detector.

- The data points included in the fit function are star-shaped. 


\section{Results (cont.)}
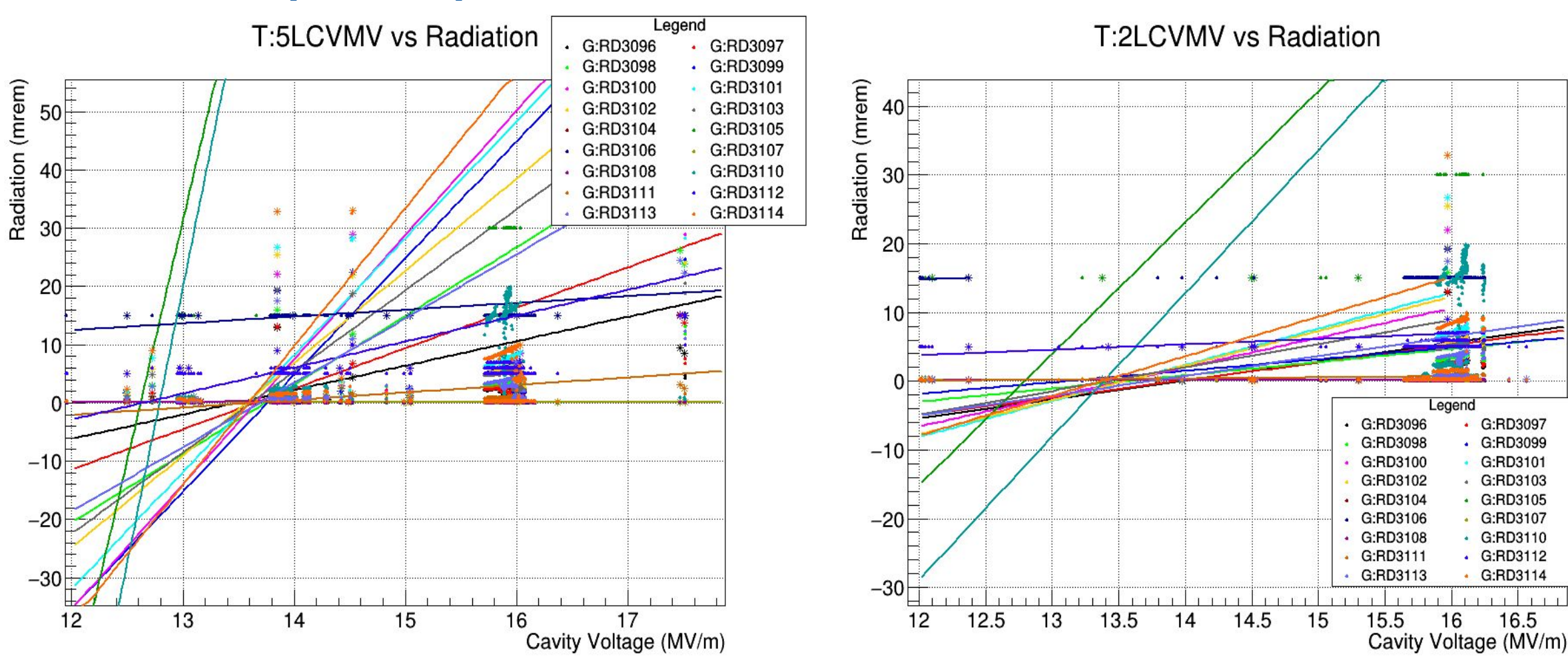

- These are the plots from the previous slide zoomed-in on the point at which (most of) the fit lines intersect.

- This intersection point is approximated as the gradient at which field emission starts to appear.

- From these plots one can conclude that the onset of field emission for cavity 5 is just below $14 \mathrm{MV} / \mathrm{m}$, and that cavity 2 has very little (if any) field emission. 


\section{Results (cont.)}

Overall Maximum values for each| device:

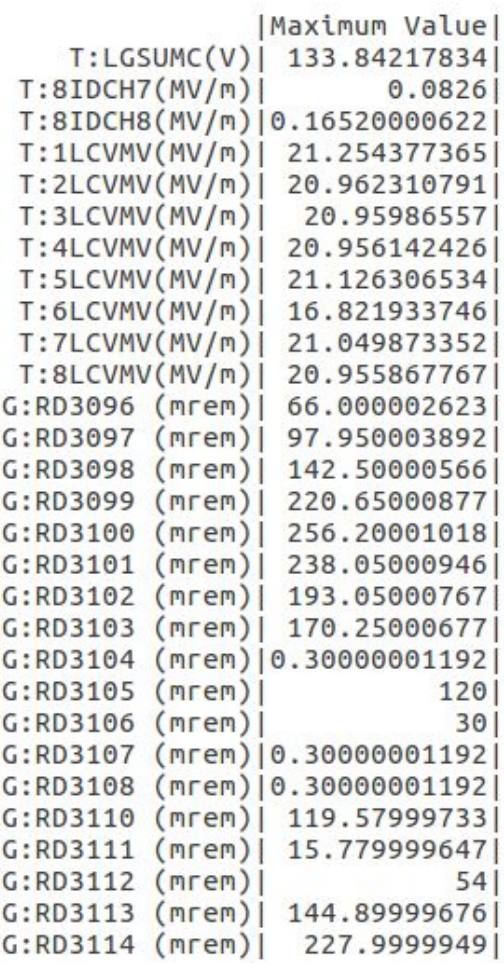

Maximum Radiation values between 15.500000 and $16.600000 \mathrm{MV} / \mathrm{m}$ for each cavity

\begin{tabular}{|c|c|c|c|c|c|c|c|c|}
\hline G:RD3096| & 12.91 & 12.9 & 12.91 & $23.4 \mid$ & 3.31 & $2.85 i$ & 31 & 3 \\
\hline G:RD3097| & 13.051 & 13.05 & 13.051 & 33.751 & 5.251 & 31 & 3.15 & 3.15 \\
\hline G:RD3098| & 15.91 & 15.9 & 15.91 & 49.81 & 6.751 & 4.051 & 4.21 & 4.21 \\
\hline G:RD3099| & 19.21 & 19.2 & 19.2 & 51.31 & 7.951 & 6.751 & 6.91 & 6.91 \\
\hline G:RD3100| & 22.051 & 22.051 & 22.051 & 35.71 & 8.551 & 8.41 & 8.551 & 8.55 \\
\hline G:RD3101| & 26.7 & 26.7 & 26.7 & 26.71 & 8.71 & 8.41 & 8.71 & 8.7 \\
\hline G:RD3102 & 25.51 & 25.5 & 25.51 & 25.51 & $6.45 i$ & $6.45 i$ & $6.45 \mid$ & 6.45 \\
\hline G:RD3103 & $19.35 \mid$ & 19.35 & 19.35 & $19.35 \mid$ & 5.851 & 5.851 & 5.851 & 5.85 \\
\hline G:RD3104| & 0.15 & 0.15 & 0.15 & 0.15 & 0.15 & $0.15 i$ & 0.15 & 0.15 \\
\hline G:RD3105 | & 120 & 120 & 120 & 120 & 301 & 301 & 301 & 30 \\
\hline G:RD3106 & $15 \mid$ & 15 & $15 \mid$ & $15 \mid$ & 15 & $15 \mid$ & $15 \mid$ & 15 \\
\hline G:RD3107| & 0.15 & 0.15 & 0.15 & 0.15 & 0.15 & $0.15 \mid$ & 0.15 & 0.15 \\
\hline G:RD3108| & $0.3 \mid$ & 0.3 & $0.3 \mid$ & 0.31 & 0.31 & 0.31 & 0.31 & 0.3 \\
\hline G:RD3110| & $119.58 \mid$ & $119.58 \mid$ & $119.58 \mid$ & $119.58 \mid$ & $19.86 \mid$ & $19.86 \mid$ & $19.86 \mid$ & 19.86 \\
\hline G:RD3111| & 1.08 & 1.08 & 1.08 & 1.08 & 1.08 & 1.08 & 1.08 & 1.08 \\
\hline G:RD3112| & 91 & 91 & 91 & $11 \mid$ & $7 \mid$ & $7 \mid$ & $7 \mid$ & \\
\hline G:RD3113 | & $17.46 \mid$ & $17.46 \mid$ & $17.46 \mid$ & 46.921 & 7.081 & 4.321 & $4.44 \mid$ & 4.44 \\
\hline G:RD3114| & 32.881 & 32.881 & $32.88 \mid$ & $32.88 \mid$ & 9.961 & 9.721 & $9.96 \mid$ & 9.96 \\
\hline
\end{tabular}

- These are screenshots of the data tables produced by the ROOT/C++ program.

- The table on the left shows the maximum values recorded by each cryomodule device.

- The right table on the right shows the peak radiation values for each voltage cavity and for each radiation detector in the range of 15.5 and $16.6 \mathrm{MV} / \mathrm{m}$. 


\section{Conclusion and Outlook}

- Cryomodule field emission and dark current Data can now be obtained mostly automatically using an ACL script and the Root/C++ program.

- The Root/C++ program can be used to analyze data from any cryomodule.

- Next steps:

- Find a more precise and objective way of finding the cavity gradient where detectable radiation starts to appear.

- Perform a more complete analysis of dark current production.

- Make a short tutorial for future users on how to use the ACL script and ROOT/C++ program. 


\section{Acknowledgements}

Thank you to:

- Elvin Harms, my mentor

- Brian Hendricks, for creating the ACL scripting language - Eric Prebys and Rosa Foote for coordinating the Lee Teng Internship 


\section{References}

[1] T. Arkan et al., "LCLS-II 1.3 GHz Design Integration for Assembly and Cryomodule Assembly Facility Readiness at Fermilab," in Proc. 17th Int. Conf. on RF Superconductivity (SRF2015) , Whistler, BC, Canada, Sep. 2015.

[2] E. Harms et al. , " Fermilab Cryomodule Test Stand Design and Plans," in Proc. 17th Int. Conf. on RF Superconductivity (SRF2015) , Whistler, BC, Canada, Sep. 2015. 


\section{Backup Slides}




\section{Results (cont.)}
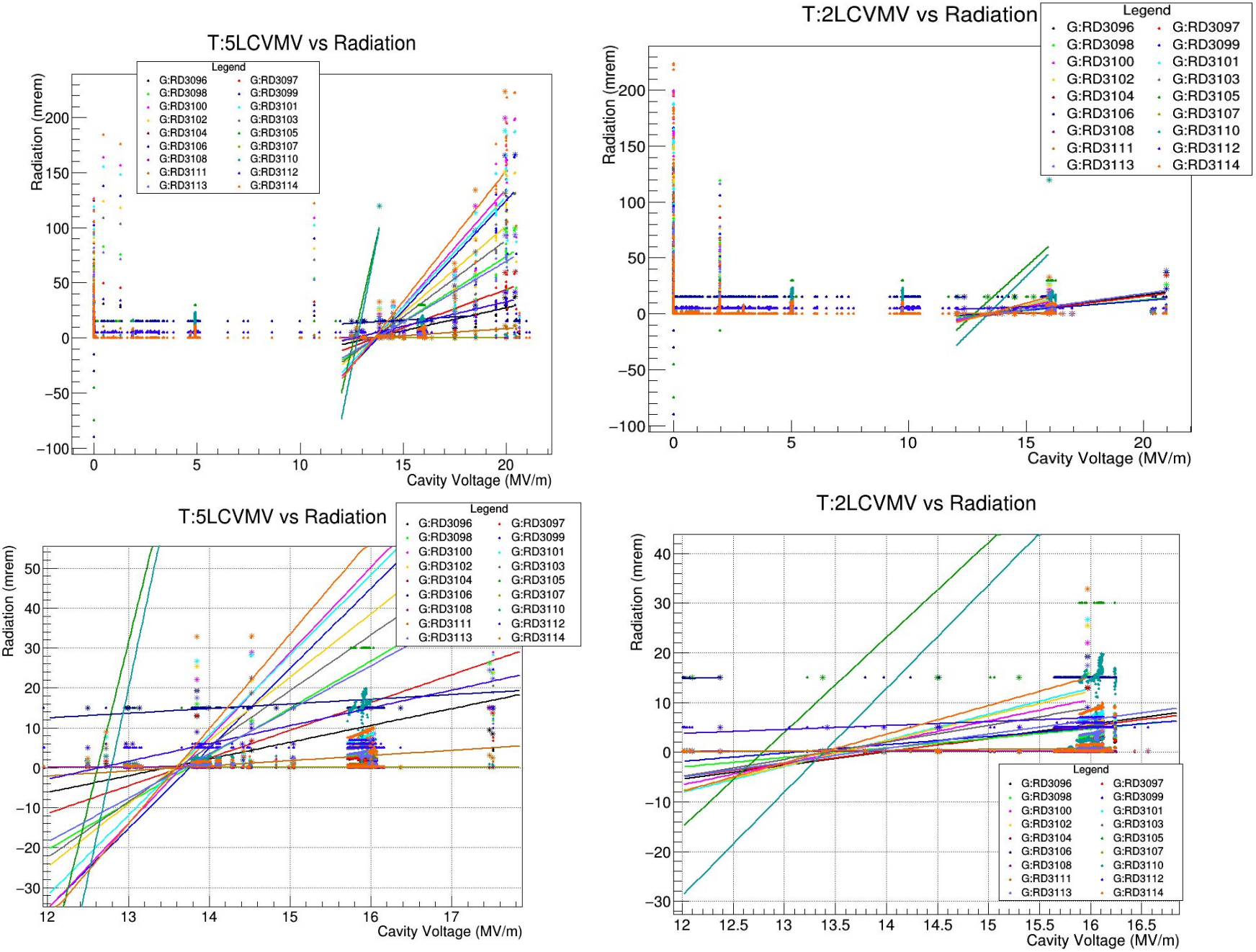

Top Plots: Full graph of cavity voltage vs radiation for cavities 5 (left) and 6 (right). Bottom Plots: Close up of the regions where the fit lines on the plots intersect. 